\title{
Las ciencias del lenguaje y el lenguaje de las ciencias
}

La Sociedad Asturiana de Filosofía, que ya organizara con éxito los dos primeros congresos en los años 1982 y 1983, comprendió pronto que la intensidad de los trabajos, la altura intelectual de las reuniones, y la abundancia de medios precisos para su celebración, requerían espaciarlas un poco más. Acordó, por ello, que los congresos de teoría y metodología de las ciencias tuvieran lugar bienalmente; correspondió así al año 1985 la organización del tercero, habido entre los días 23 y 28 de septiembre con la asistencia de cerca de quinientos congresistas.

Un gran «leitmotiv» se propuso para esta edición: «Las ciencias del lenguaje y el lenguaje de las ciencias». Es indudable que, como punto de partida, resulta muy sugerente este lema; pero no lo es menos que su generalidad entraña un serio riesgo de imprecisión en el tratamiento. Sin prejuzgar la calidad de las intervenciones que más adelante reseñaremos, puede asegurarse que este tercer congreso adoleció de una mayor inconcreción que los precedentes $y$, en consecuencia, de una menor vivacidad en los debates.

Acudieron a la convocatoria de esta reunión varios notables especialistas extranjeros, como Kenneth Pike, Abraham Moles, Janos Kelemen, René Thom, o Karl Otto Apel. El desconocimiento por casi todos ellos del idioma español hizo necesario el uso de traductores simultáneos. Cierta falta de costumbre de la mayoría asistente de participar en convenciones internacionales, la dificultad que la traducción simultánea acarrea a la controversia, y la discutible calidad de esa traducción, contribuyeron también a obstaculizar la fluidez de los coloquios.

De forma paralela a la celebración del congreso, tuvo lugar el primer encuentro de estudiantes de filosofía, al que acudieron alumnos de distintas universidades españolas. En él, integrado como una sección del congreso, se debatieron cuestiones relacionadas con la filosofía de la ciencia en sus nuevas 
perspectivas, así como sus implicaciones en la enseñanza media y universitaria. Se espera que en los comienzos del año 1986 se publiquen las actas de este encuentro estudiantil (no habiéndose anunciado, en cambio, la edición de actas del congreso «grande», a diferencia de lo ocurrido con los dos anteriores).

Terminamos estas referencias previas, dedicadas a los aspectos organizativos y sociales del congreso, señalando que sus sesiones tuvieron lugar esta vez en Gijón - y no en Oviedo- en las magníficas instalaciones del Centro de Enseñanzas Integradas (lo que fuera sede de la Univerdidad Laboral de Gijón). El acto inaugural, celebrado en la mañana del 23 de septiembre, contó con representaciones oficiales diversas, destacando la máxima autoridad municipal, y el Consejero de Educación y Cultura del Principado de Asturias, que viene colaborando con la Sociedad Asturiana de Filosofía en muchas de sus actividades. En este acto se resaltó la importancia de estas reuniones interdisciplinarias de filósofos y científicos, aunque sean promovidas por filósofos (a quienes, según Gustavo Bueno, corresponde esta función de crítica epistemológica).

A excepción de la última jornada, en cuyo mediodía se cerró el congreso, las sesiones se produjeron por la mañana y por la tarde. Las matinales fueron ocupadas por las ponencias y coloquios subsiguientes, ocupando las vespertinas las comunicaciones y mesas redondas.

Aclaremos, antes de seguir adelante, que bajo el lema de ciencias y lenguaje mencionado antes, se concedió especial atención a dos disciplinas -matemáticas y lingüística- siguiendo la costumbre implantada en los anteriores congresos. Es cierto, sin embargo, que la representación de matemáticos y filósofos de la matemática fue más numerosa que la de lingüistas y filósofos del lenguaje, como podrá apreciarse en el resumen que sigue.

1. La primera ponencia corrió a cargo del matemático francés René Thom. La obra de éste ha causado, en los últimos años, un gran impacto en la comunidad científica por su enunciación de la llamada teoría de las catástrofes; se trata (en «Estabilidad estructural y morfogénesis») de una aplicación del formalismo matemático a la explicación de las rupturas que la ontología describe.

En su intervención, el profesor Thom abordó la pertinencia de la noción de causa en las ciencias. Las corrientes positivistas, que en tantos aspectos han reclamado su entronque en la tradición empirista, criticaron - por considerarla un residuo metafísico científicamente inadmisible - la presencia de causas en las verdaderas ciencias; de acuerdo con esta posición, las ciencias deberían poner leyes allí donde antes se hablaba de causas. 
Desde la perspectiva de su disciplina, entiende que hay un campo de formas en el espacio, destacadas sobre el fondo, cuyas connotaciones deben ser descritas. Aun cuando Hume y sus partidarios atribuyen al mero hábito la fijación de tales conexiones, Thom entiende que para la ciencia contemporánea es muy útil la noción de causa siquiera por motivos pragmáticos, pues el reemplazo de las causas por leyes exigiría una compleja labor, ante el elevado número de leyes que serían precisas.

El concepto aristotélico de causa puede, pues, ser re-introducido. Y puede serlo en sus cuatro acepciones originales, formal, material, eficiente y final. El ponente sostuvo, para justificar su propuesta, que hay una ontología causalista implícita en los lenguajes naturales, que también han resultado ser ineliminables del panorama de las ciencias: los nombres y los verbos, en efecto, designan en esos lenguajes entidades que hacen alguna cosa, que llevan algo a efecto.

El recurso a la causa formal brinda la posibilidad de suministrar un modelo matemático a determinados procesos puramente fenoménicos; citando a Laplace, se sostiene como posible la descripción de trayectorias en un espacio. Junto a ella, la causalidad material es ineludible, pues incluso en la ciencia considerada formal por excelencia, la matemática, hace falta un principio del cual partir. La causalidad eficiente carece de modelos matemáticos, aunque podría encontrarse en el esquematismo cibernético (a la manera de $« A \longrightarrow B »$, por ejemplo, es decir «A funciona sobre $B » . .$.$) . Por último, tampoco la causa-$ lidad final es prescindible, a pesar de sus connotaciones metafísicas: en tal sentido es llamativa la observación hecha por Thom declarando, como uno de los dos más grandes problemas de la ciencia actual, el de la emergencia de la finalidad biológica.

La tesis del ponente, una vez sentada la conveniencia de recuperar para el lenguaje científico el empleo de causas, es que la causa eficiente y la formal deben ser reunidas; el control de los fenómenos requiere el paso de la causa eficiente a la formal. Para ejemplificar esto se hizo una representación gráfica -al modo de las funciones lógico-matemáticas - de un caso posible de causalidad eficiente: «el calor funde el hielo». Admitiendo que, cuando hay dos entidades, un agente produce transformaciones sobre otro, el propuesto fenómeno de fusión sería formalizable paralelamente al incremento de la temperatura.

En el coloquio posterior a su intervención, precisaría el orador que la causalidad general no es necesariamente binaria, sino que lo normal es que -a pesar de que los científicos se expresen habitualmente en términos diádicos como si cada efecto tuviera una sola causa - en la realidad se dé un alto número de concatenaciones. 
Para René Thom, toda ontología que pretenda ser inteligible ha de contemplar entidades resaltantes en las que puedan hallarse propiedades pregnantes. Su concepto de pregnancia, que podría situarse para el plano del lenguaje en la función predicativa, es una adaptación del que a principios de siglo popularizase la psicología de la «Gestalt». Su importancia teórica es grande por cuanto permite salvar la más tradicional dificultad de la causalidad (la invisibilidad del hecho causal mismo), mediante la creación de un campo de interacción entre causa y efẹcto.

Como puede imaginarse por lo dicho hasta aquí, la exposición de Thom constituyó un original principio, especialmente por venir la reivindicación de la causalidad aristotélica de un matemático tan prestigioso.

Entre las comunicaciones del día destacaremos la de Julián Velarde, «el lenguaje de la lógica», en la que analizó las teorías del significado de algunas importantes construcciones lógicas: Aristóteles, los estoicos, la escolástica, Peirce, y Frege. El comunicante sostuvo que la significación es una función, es decir el conjunto de transformaciones operadas sobre el lenguaje, entendiendo que la estructura del significado no es dual, como acostumbra a pensarse, sino trial o triádica. Agrupando los elementos en tres clases - al decir, por ejemplo, de Peirce: la de los objetos, la de los signos, y la de los interpretantes - se concebirían los de la última clase como resultado de las operaciones entre los elementos de las dos primeras.

M. ${ }^{\text {a }}$ Victoria Carreras Arch presentó una comunicación, junto con Ángel Blanco, sobre «Ética de la investigación y de la ciencia en psicología». En ella se trataron los problemas que plantea la experimentación psicológica y sobre los que, a pesar de los diversos códigos deontológicos promulgados en los últimos cuarenta años, se mantiene la duda acerca de la moralidad o inmoralidad del engaño en los experimentos. Los psicólogos defendieron, desde el rechazo del engaño como algo no-ético, el respeto de la dignidad y la libertad de los individuos como imprescindible actitud en las investigaciones de psicología. Por más obvio que esto pueda parecer, resulta necesaria la insistencia ante la proliferación de prácticas experimentales con humanos.

2. El filósofo alemán Karl Otto Apel abrió la segunda jornada con una disertación titulada «Linguistic meaning and intentionality». Desde una posición doctrinal muy próxima a los presupuestos frankfurtianos y a la hermenéutica alemana del lenguaje, Apel analizó los tres grandes giros obrados en el terreno del lenguaje de este siglo. Se habría dado, en primer lugar, un giro lingüístico impulsado por el «Tractatus logico-philosophicus» de Wittgenstein, 
caracterizado por la falacia abstractiva que él atribuye al semanticismo transcendental. Tras ése, un segundo giro -identificable acaso con la llamada filosofía del lenguaje ordinario- habría sido el pragmático de la teoría general del significado; su rasgo más destacado, el intento de superación de la aludida falacia abstractiva. Por último, un giro al que cabe denominar psicologístico (por haber sido propugnado por una cierta filosofía psicologista, con John Searle a la cabeza), habría pretendido la reducción del significado al plano de las intenciones prelingüísticas.

Apel, partidario de una recuperación de una nueva filosofía en la creación de un campo ético-comunicativo común - lo que él llama comunidad de comunicación-, afirma que ante el giro psicologístico, que parece excluir el profundo ámbito de la racionalidad, debe defenderse un giro lingüístico que pase por la aceptación de un principio: el de no transcendentabilidad de la forma sintáctico-semántica del lenguaje.

La comunicación efectiva, como un hecho real entre los individuos, debe recuperar su papel como gran función del lenguaje, una vez desarticulado el presunto isomorfismo lenguaje-realidad apuntado en el «Tractatus».

A continuación intervino Abraham Moles, pensador original y difícilmente encuadrable por sus siempre sugerentes reflexiones sobre la percepción, la psicología de masas, la epistemología, o la sociología de la vida cotidiana. Por esa su familiaridad con las ciencias humanas resultó más atractiva su exposición sobre «Le langage des sciences sociales: un traitement rigoureux des phenomènes imprécis». En ella trató de la metodología de esas ciencias de lo impreciso, lo borroso, o lo vago.

Moles, al tiempo que deja constancia de la extendida creencia de que la matemática no puede aplicarse a las ciencias humanas (por ocuparse éstas de fenómenos vagos), advierte que no hay motivo para sostener esa creencia: el que los hechos sociales objeto de esas ciencias sean vagos o imprecisos no justifica que su tratamiento haya de ser igualmente borroso o carente de precisión. El error que subyace a esa extrapolación procede, al entender del ponente, de la frecuente confusión que en los matemáticos se da entre formalizar y cuantificar, como si esto último fuese el único objetivo de las ciencias.

Para salvar ese obstáculo, Moles cree que hay que introducir en epistemología un concepto a menudo olvidado: el de fidelidad. Marcar como objeto científico la fidelidad a la experiencia, es decir el reconocimiento de una cierta regularidad en los hechos de la naturaleza, es algo mucho más importante y productivo que ceñirse al logro de la precisión.

Moles expuso algunas consideraciones sobre las ciencias humanas en la actualidad, antes de formular sus propias propuestas metodológicas. A su parecer, cualquier epistemología de las ciencias sociales debe reconocer que éstas 
se ocupan de fenómenos por esencia imprecisos. Ello no justifica la arbitrariedad, pues «el pensamiento de lo vago no es igual que la vaguedad en el pensamiento»: en las ciencias sociales se da un principio de incertidumbre pragmático, que evita la frecuente confusión entre el rigor y la precisión, y que permite que esas ciencias se construyan sobre las nociones de regularidad y predictividad como conceptos epistemológicos primordiales. Por último, advirtió que, si es verdad que la lógica científica ha perseguido definir los objetos en tanto se presentan como fenómenos, no es menos cierto que éstos, los fenómenos, son creados por la escala que se les aplica.

Sentadas estas apreciaciones en torno a las ciencias de lo vago o impreciso, Abraham Moles detalló los grandes principios que podrían vertebrar una metodología de lo «vago», de entre los que recogemos los más señalados. 1) Preeminencia del ordinal sobre el cardinal, pues es más significativo analizar los factores latentes en las matrices ordinales que ocuparse de acumular meras cifras. 2) Jerarquización de los objetos, que permita segregar lo principal de lo accesorio, en línea con la oposición dialéctica que la «Gestalt» descubrió entre forma y fondo. 3) Prioridad de la evaluación sobre la medida, lo que exige crear escalas diferenciadas por grupos. 4) La fidelidad de una medida crece en razón inversa a su precisión. Esto se complementaría con el establecimiento de ecuaciones simbólicas, concediendo una mayor importancia a las relaciones entre medidas que a las medidas mismas, (aplicando matrices de similaridad e interacción). 5) Finalmente, constitución de modelos explicativos («pensar es esquematizar»), y crítica repetitiva de su funcionamiento.

La intervención de Moles fue particularmente importante por la originalidad de sus trabajos como psicólogo y sociólogo: hacer uso de la cibernética, y de los métodos estadísticos y matemáticos, no puede constituir nunca el objetivo terminal de las ciencias sociales o ciencias de los fenómenos imprecisos. Es más rentable en estas ciencias ser fiel a los hechos que expresarlos con exactitud.

José A. Díez Calzada, en su comunicación sobre la noción de progreso científico en los epistemólogos estructuralistas (Sneed, Stegmuller, Moulines), destacó la distancia que la concepción estructuralista de las teorías marca entre teorías científicas, irrefutables, y aserciones empíricas, refutables por poder ser declaradas verdaderas o falsas. El estructuralismo entiende el progreso de la ciencia como derivado del dinamismo de las propias teorías, y no en relación a un paulatino acercamiento a la verdad. Queremos dejar constancia aquí de que el estructuralismo, poco representado en este congreso, sigue resultando asunto polémico - ténganse presentes las disputas entre los sneedianos y sus críticos- por lo que las epistemologías realistas califican de excesivo conceptualismo, de teoreticismo puro. 
Julián Garrido, físico que ya evidenció en anteriores congresos su interés por la teoría de su ciencia, presentó una comunicación sobre «Posibilidad física y posibilidad técnica» que contribuyó, a nuestro parecer, al esclarecimiento de algunos conceptos confusamente empleados a menudo. Según Garrido, la posibilidad física pertenece al campo material, factual; la posibilidad técnica, por el contrario, está referida al campo objetual, y ha de pensarse siempre en orden a fines determinados. La clave del asunto - ejemplificado con un teorema de la termodinámica, el de Carnot- estriba en que la física se opera con leyes, que no tienen carácter normativo sino meramente factual, en tanto en la técnica se opera con reglas. Las leyes físicas obedecen a fines teóricos, y no a fines pragmáticos: no deben ser confundidas, por tanto, con las leyes del derecho como hacen, por ejemplo, quienes asocian el problema de la libertad humana al del determinismo o indeterminismo físicos.

Alberto Cardín presentó una comunicación sobre la elaboración de teorías antropológicas bajo el llamativo título de «El efecto Rashomon en etnología». Para Cardín, pretendiendo criticar la antropología de Marvin Harris, el caso Rashomon (novela llevada al cine por Kurosawa, en la que cinco testigos ofrecen una versión distinta de un mismo suceso) es metafórico de lo que ocurre en etnología: en ésta los datos no son contrastables empíricamente, sino sólo textualmente, por lo que están sometidos al «efecto Rashomon», es decir a la pluralidad y disimilitud de testimonios e interpretaciones. Ante la perplejidad que tal efecto produce, Harris defiende la perspectiva «etic», instalada en la cultura propia del investigador y no en la cultura investigada. Frente a ello, Cardín propone un «status» dialéctico de la etnología o antropología, entendiendo aristotélicamente lo dialéctico como discurso de lo problable - ante las distintas versiones textuales-, y respetuoso con otras culturas. En el fondo, la crítica de la perspectiva «etic» adoptada por Harris encierra el rechazo del totalitarismo uniformador que oculta el punto de vista etnocéntrico de la única cultura que hace antropología: la cultura occidental.

3. La figura de Kenneth L. Pike escapa a cualquier clasificación convencional. Si bien representa una de las dos más importantes líneas en la lingüística contemporánea - la que pudiera llamarse sociolingüística, para contradistinguirla de la generativo-transformacional encabezada por Noam Chomsky-, sus investigaciones han generado interesantes sugerencias para la filosofía del lenguaje; además, los estudios realizados por él en multitud de culturas idiomáticas distintas han suscitado relevantes observaciones antropológicas. La personalidad de Pike se ha convertido, así, en un paladín del diálo- 
go igualitario entre las culturas, a partir curiosamente del análisis de las formas del diálogo, hasta el punto de haber sido nominado en alguna ocasión como candidato al premio Nóbel de la paz.

$\mathrm{Su}$ conferencia, "The relation of language to world view» (Philosophy), fue un alegato en favor de una ciencia del lenguaje incardinada en las tramas sociales y culturales en que los idiomas se desenvuelven. Conviene recordar que Pike se mueve en la estela teórica de Edward Sapir y Leonard Bloomfield, $\mathrm{y}$ de todos los que han sostenido que el lenguaje y el pensamiento tienen una raíz fundamentalmente socio-cultural. De su trabajo como lingüista surgió la distinción «emic»/ /etic», distinción que casi se ha convertido en canónica entre antropólogos y filósofos del lenguaje; aunque originariamente esos clichés servían como abreviaturas del par filológico fonemic/fonetic, su desarrollo ha llevado a su consolidación como nociones teóricas complejas. Se entiende así que es «emic» aquella estructura que se sitúa dentro de un sistema (como pudiera ocurrir con la gramática), mientras se considera «etic» —en el mismo sentido en que lo utilizábamos antes al reseñar la crítica de Alberto Cardín a Marvin Harris- a toda estructura emplazada fuera de ese mismo sistema, (p.ej. los sonidos que se emiten).

K.L. Pike entiende que la investigación científica de las lenguas exige una suerte de aceptación previa, y dice perseguir una actitud filosófica integradora a partir de su pluriforme experiencia lingüística, de conocedor de lenguas. Se trata, pues, de desenmascarar las verdaderas consecuencias de la obra chomskyana: Chomsky habría desencadenado una revolución más filosófica que linguística, puesto que ha reemplazado el interés en la diversidad de las lenguas por el interés en los universales presuntamente innatos.

Dado que en las lenguas hay aspectos o sonidos «emic» conectados muy directamente con un ambiente cultural concreto, con ciertas características humanas innatas o, simplemente, con el entorno inmediato de las gentes, el observador que se relaciona con los datos lingüísticos termina por ser parte de los datos cognoscibles mismos. En este sentido, Pike piensa con Kant que, frente a los noúmenos, los fenómenos suponen una determinada manera de conocer el objeto por parte del sujeto. Por este motivo, critica a quienes, como Jerrold J. Katz, sostienen desde una perspectiva platónica que el lenguaje es una entidad abstracta, independiente de la sociedad, e inmutable.

La aceptación del lenguaje como jerarquía entraña consecuencias filosóficas. Debe tenerse en cuenta que no existen sonidos aislados y que, por tanto, las unidades lingüísticas ocurren en unidades «émicas» más amplias, enclavándose en tres tipos diferentes de jerarquía: referencial o léxica, gramatical, y fonológica. Pero hay otro nivel más que excede en complejidad al fonético, y es la interacción social. Según Pike, el entendimiento de cualquier conversa- 
ción exige profundizar en esa interacción social, por lo que resulta inaceptable el reduccionismo chomskyano que pretende poder descifrar los discursos sin intercalarse en el tejido de la sociedad que los maneja.

Naturalmente, aquí se está defendiendo un cierto relativismo o perspectivismo lingüístico-cultural. Por una parte, dado que las palabras son, como la vida, multidimensionales; es decir, no son lógicas ni lineales, pues sabemos imposible el viejo ideal del matemático y lógico Alonzo Church de que a cada palabra corresponda un solo significado. Por otro lado, puesto que los observadores crean en alguna forma su propio mundo («émico»), del mismo modo que la cosmovisión de cada cultura encierra sus propios aspectos émicos.

El profesor K.L. Pike completó su exposición mostrando su modelo «tagmémico», paradigma que, mediante cuatro grandes categorías, posibilita una plantilla normalizada de análisis de sentencias lingüísticas (aceptada, por ejemplo, en la tarea de traducir la Biblia que está realizando la institución de lingüistas que el propio Pike preside).

La filosofía de los países de la órbita soviética tuvo en el húngaro Jano Kelemen a su único representante en este congreso. Su intervención, titulada «György Lukács y la problemática de la lengua», fue dedicada a la filosofía del lenguaje del impulsor de la escuela de Budapest en el primer centenario de su nacimiento. Fiel a ese propósito, la ponencia de Kelemen fue menos una visión original del lenguaje desde la óptica marxista que una revisión del tratamiento lukacsiano.

Marx y Engels, en «La ideología alemana», analizaron la significación de las palabras en un doble plano, personal y mercantil. Para ellos, las relaciones de clase y las ideologías - particularmente la de la burguesía - están enclavadas en el lenguaje y en el uso que de él se hace. Una eventual modificación de las circunstancias sociales, entre el tiempo de Marx y el nuestro, no elimina la problemática de la naturaleza social del lenguaje, pues en todo caso el lenguaje surge de una necesidad humana de intercomunicación...

El objetivo de Lukács fue investigar las relaciones que se producen entre el lenguaje y la ideología de la clase dominante. En opinión de Kelemen, ésa sigue siendo una tarea crucial para el marxismo de nuestro tiempo.

La historia del marxismo, por lo que a este asunto respecta, estuvo durante muchos años dominada por la doctrina fisiológica de Pavlov. De acuerdo con éste, y en expresión ya consagrada, el lenguaje sería un segundo sistema de señales, construido por encima de un primer sistema, el sensorial. Una de las más extensas e influyentes obras de Lukács, la «Estética», vino a modificar esa-postura de tinte pavloviano: Lukács introduce allí una especie de sistema intermedio de señales, entre el primario y secundario, que atiende más hondamente a las relaciones entre signos y pensamiento $\mathrm{y}$, sobre todo, entre 
trabajo y habla. A diferencia de Pavlov, Lukács concede una gran importancia a la indagación histórico-genética para el conocimiento de los fenómenos (y también, como es obvio, de los fenómenos lingüísticos).

Para el ponente, la génesis del lenguaje tiene como gran elemento impulsor al trabajo, a la mano de obra. La misma estructura del lenguaje - sin ser isomórfica con las relaciones de producción, pues la lengua tiende a individualizarse, a autonomizarse- surge del trabajo como actividad. Si se considera esto, y se rectifican (como pareció apuntar el propio Lukács en su «Ontología») las simplistas identificaciones, entre palabras y objetos, que había en su obra primera, los planteamientos de éste son aún aceptables. Entre la complejidad social, el lenguaje constituye para Kelemen un muy importante complejo.

En la tarde de este día 25 se celebró una mesa redonda sobre el tema genérico «Comunicación y lenguaje», de la que formaron parte los profesores Apel, González Escribano, Pike, Kelemen, Cardín, y Moles. Ejerció como moderador el conocido periodista asturiano Juan Cueto Alas, personaje para quien resulta singularmente propicio el reciente calificativo de «comunicólogo», en atención a su interés por los modernos medios de comunicación de masas y por el aparato tecnológico que los sustenta e inspira. Aunque en otras ocasiones hemos dejado constancia de la escasa fertilidad de las mal llamadas «mesas redondas» -incluso cuando quienes las forman acrediten una talla intelectual tan infrecuente como la de los mencionados - vamos a anotar la idea central recogida en la intervención de cada uno de ellos.

Para K.O. Apel el lenguaje es, recordando a Humboldt, un vehículo para todo. La comunicacional es, pues, la más importante de todas sus funciones, dado que el pensamiento humano tiene una estructura interna dialógica. Cuando se piensa, incluso aislada o silenciosamente, se celebra una especie de diálogo con uno mismo. Comunicar es, así, algo inseparable de la articulación del lenguaje. Y la comunidad de comunicación a que más arriba aludimos se convierte en un importante fundamento de la ética, de la ética del discurso social.

El profesor González Escribano, especialista en filología inglesa de la Universidad de Oviedo, matizó algunas de las anteriores afirmaciones. A su entender, el lenguaje no tiene una función meramente comunicativa; y ello por un doble motivo. Primero, porque el concepto de comunicación es tan amplio y heterogéneo que no es científicamente abarcable. Segundo, porque, si se atendiese solamente a las exigencias que la comunicación plantea, quedarían sin explicar muchas transformaciones en el lenguaje, así como muchos aspectos de su estructura profunda.

K.L. Pike insistió en la importancia que la interacción social tiene: tanta 
que la vida y el pensamiento normales serían imposibles sin ella, y hasta pensar en uno mismo resultaría inviable. El mecanismo que esa interacción social exige necesariamente es el lenguaje, el lenguaje como medio de comunicación inherente a la vida social. La lógica formal y las matemáticas, aun cuando pueden ser utilizadas por la lingüística, constituyen formas muy posteriores al lenguaje en sociedad. Como observó Pike, todos los pueblos se comunican, pero no todos ellos hacen lógica o matemáticas.

J. Kelemen, conectando con su exposición matinal, acudió también al que muchos consideran primer filósofo del lenguaje, Guillermo Humboldt. Aunque éste relegase la comunicación como función lingüística primordial, reconoció el lenguaje como relacionado con el trabajo, trabajo duradero del espíritu. Si se recupera la noción de trabajo, en línea con las preocupaciones de Marx y Engels por el lenguaje, habrá de reconocerse que la comunicación forma parte de la historia de las ideas y de la historia real. Y una ojeada a esa historia muestra que, al menos en la tradición europea, el lenguaje se señala como medio principal de comunicación: desde Descartes hasta la filosofía transcendental y a la filosofía germánica más reciente.

Disintiendo de sus compañeros de mesa, A. Cardín sostuvo que el reconocimiento del hecho del lenguaje, y de que se produce la interacción social, no implica admitir que haya comunicación. Para apoyar su tesis, acudió a la teoría de la sexualidad de Jacques Lacan: para éste, entre las personas no se establecen relaciones sexuales, aunque sí se celebran actos y gestos sexuales que mediante equívocos transmiten significados. Cardín cree que hay que perseguir una comunicación efectiva, directa y no elíptica; pero afirma que nos desenvolvemos en un gran simulacro, en una cadena de equívocos a través de los que se significa algo. Como anécdota ejemplar de su pensamiento, refirió cómo en la conquista española de las Indias la toma de una nueva plaza iba seguida de la lectura de una compleja requisitoria jurídica redactada en castellano (evidentemente, la única comunicación no derivaba de la comprensión -imposible- de aquel texto, sino del estado de violencia circundante).

A. Moles, que cerró las intervenciones, tampoco se mostró optimista sobre las posibilidades de comunicación en la sociedad de nuestro tiempo. A los muchos tipos de comunicación existentes pertenece un rasgo común: se trata de procesos que exigen una cierta complicidad de un término a otro mediante canales comunicadores. Lo que en esta época ocurre es que la importancia de lo que va a comunicarse ha quedado rebasada por la del canal a través del que se comunica. En la sociedad de comunicación de masas tiene más importancia el continente que el contenido, el canal que el mensaje.

El presidente de la Sociedad Asturiana de Filosofía, Alberto Hidalgo, presentó una comunicación sobre los contextos determinantes de la teoría de 
la ciencia. El concepto de contexto determinante, tomado del pensamiento de Gustavo Bueno, tiene el interés de permitir la comprensión del desarrollo de las teorías de la ciencia. La historia de la teoría de la ciencia, disciplina ésta que constituiría la última "capa geológica» de la filosofía, muestra que es el mismo desarrollo científico quien vitaliza las epistemologías (lo que explica, acaso, el máximo auge alcanzado por ellas en el segundo tercio del siglo XIX). Para Hidalgo, a menos que incurran en la irresponsabilidad de independizarse totalmente de la filosofía, las ciencias deberán vertebrarse en una teoría de la ciencia, incorporándose así a la modificación de las condiciones reales de la existencia humana.

4. El jesuita Alberto Dou, interesado en complementar sus investigaciones como matemático con el estudio de la fundamentación y la historia de la matemática, disertó sobre «Los lenguajes de la matemática y la mecánica teórica clásica» con el rigor y amenidad didáctica que ya evidenciara en el primero de estos congresos. En su opinión, la conexión que el congreso propone entre el lenguaje de las ciencias y las ciencias del lenguaje es muy remota, y parece difícil de establecer.

Comenzó presentando una clasificación de los lenguajes en tres grandes tipos: el de las matemáticas formales, ẹl de las matemáticas clásicas, y el de la física clásica. El primero de estos lenguajes incluiría como subtipos el de la lógica, el de la metalógica o metalenguaje de los lógicos, y otros varios posibles a partir de distintos axiomas. El segundo englobaría como primer lenguaje el de los matemáticos intuicionistas y, también, otros desde varios axiomas. Finalmente, el lenguaje de la física se presentaría en tres modalidades diferentes, el de la física clásica, el de la mecánica física, y el de la ingeniería mecánica.

Para Dou, en la matemática actual se ha superado la moda logicista impulsada por Russell, se ha aplacado el furor formalista desencadenado por Hilbert, y se ha comprobado también la inviabilidad del intuicionismo - que, de seguirse, obligaría a abandonar la mayor parte de lo hecho hasta ahora por las matemáticas-. Ante esta situación, en la que la famosa crisis de fundamentos parece haberse abandonado sin encontrar una solución definitiva, los matemáticos de nuestro tiempo asumen hallarse como en las matemáticas clásicas: en una ciencia que tiene que ver con la realidad aun cuando sean ideales las entidades con las que opera. Por este motivo, el matemático de hoy puede desenvolverse tanto en un lenguaje del primer tipo reseñado como en uno del segundo.

A modo de ejemplo de ese lenguaje, intermedio entre el de las matemáticas clásicas y el de las formales, Dou propone el sistema «NBG» (tomado de 
los nombres de los matemáticos von Neumann, Bernays y Gödel); es ése un sistema equivalente al de Zermelo-Fraenkel, que implica la reducción de la matemática a la teoría de conjuntos, y que consta de siete axiomas formados a partir del predicado de pertenencia (...es elemento de...).

Ocurre que lo dicho no es aplicable en exclusiva a las matemáticas, puesto que también la física trata de la realidad de una forma idealizada. Con ello, los objetos de la matemática clásica y los de la física teórica vienen a coincidir, situándose todos ellos en el platónico mundo de las ideas o en el tercer mundo de Popper. Para ejemplificarlo, el conferenciante presentó un sistema de la mecánica de partículas que se formaría con seis axiomas, tres cinemáticos y tres dinámicos: el modo de operar en ese sistema es muy similar al del sistema matemático «NBG» citado.

Esto no quiere decir que matemáticas y física deban ser confundidas, sino que pueden manejar lenguajes muy próximos. Hay una diferencia importante entre ambas disciplinas, que radica en su diferente resistencia a las revoluciones: mientras la física experimenta grandes revoluciones al acudir a la realidad con sus descubrimientos teóricos, las matemáticas no están sometidas a revoluciones, pues asumen todos los hallazgos teóricos sin mayor complicación. Buen ejemplo de esto es la geometría hiperbólica: la matemática no encuentra problemas en admitir, sin contradicción tan siquiera, la geometría de Euclides y la de Riemann (mientras que en la realidad física sí se produce un choque al aceptar más de una paralela por un punto dado).

El profesor Dou expuso, a continuación, algunos casos de formación de conceptos y postulados como producto de la dinámica de los lenguajes científicos: así, la larga génesis del concepto matemático de función, o los tratamientos históricos distintos dados al viejo problema físico del movimiento local.

Por último observó la escasa influencia que la teoría de las revoluciones científicas, de T.S. Kuhn, ha ejercido en las matemáticas, a diferencia de la física. Para Dou, la aportación kuhniana de la problemática social a la fundamentación de las teorías científicas puede ser positiva si se aprovecha -como K.O. Apel ha sugerido en su pragmática transcendental- para buscar un fundamento transcendental, una base transcendente, a la matemática.

Ricardo Sánchez Ortiz de Urbina, recién incorporado a su cátedra de filosofía en la Universidad de Valladolid, presentó en este congreso un complejo análisis epistemológico-histórico-filológico, titulado «algunos aspectos del lenguaje de la matemática griega: los 'porismas'». La exposición se centró, con la reconstrucción de la historia de la teoría de la ciencia como objetivo, en el concepto de «porisma». Este término, cuyo origen etimológico le vincula con «riqueza», por un lado, y con «paso» o «vía hacia algo», por otro, fue 
frecuentemente utilizado en la matemática griega para significar «corolario»; si éste fuera su único significado, no merecería mayor atención, pues el sentido de recapitular lo anterior (no aportando nada nuevo) es irrelevante para la epistemología.

Hay, sin embargo, una significación distinta, de lo que son los «porismas» («porismata»), presente en Euclides y Diofanto: se trata, para ellos, de teoremas insuficientes, mucho más próximos al rigor material —que Urbina vincula con la sensación táctil- que al rigor formal - visual-. La cuestión que en el fondo se ventila es la del rigor matemático, y presumiblemente la del rigor científico en general: pues se sabe que, por lo común, el salto de las barreras y la ruptura de restricciones redundan en beneficio de la creatividad del investigador.

En la geometría griega se reconocían tres métodos: planos, sólidos y lineales. Los planos, que son los más teóricos y los más claros, presentan el inconveniente de ser más restrictivos, pues en ellos se construye con regla y compás. Según el ponente, esto es lo que define las exigencias metodológicas antiguas. Para él, el rigor antiguo se centra en la geometría, y está inspirado en una filosofía del realismo de la expresión (de ahí el empleo instrumental del compás y la regla); en cambio, el rigor moderno concierne a la aritmética, inspirándose en una filosofía del operacionalismo de la deducción (como la historia de la matemática moderna y contemporánea parece atestiguar).

La distinción entre problemas y teoremas es de interés porque aquéllos son cuestiones totalmente abiertas, una vez satisfechas sus necesarias condiciones de posibilidad, mientras los teoremas alcanzan un nivel demostrativo concluyente. Es ahí donde los porismas tienen un lugar, en tanto se distinguen de los problemas pero no alcanzan aún la calidad de teoremas. De esa forma, el estilo porismático da paso a una perspectiva científica singular, constituyéndose en un motor valiosísimo del progreso en la historia de las matemáticas por su status intermedio, ni problemático ni teoremático. En ese sentido, es interesante recordar que Euclides escribió un libro de porismas que, si bien se ha perdido, puede ser reconstruido mediante técnicas filológicas que prueban su carácter de obra euclidiana más fecunda y creadora para la geometría.

Sostuvo por último el ponente que la necesaria oscuridad de los porismas faculta para, desde una visión histórica retrospectiva, conectar en una misma saga matemática a Euclides con Fermat, Gauss o Riemann.

El trabajo del profesor Sánchez O. de Urbina será uno de los más beneficiados por una eventual publicación de las actas del congreso, ya que ésta permitiría captar en toda su dimensión los múltiples matices filosóficos y filológicos acumulados en su intervención oral.

Entre las comunicaciones de la tarde merecen citarse dos. La del helenista 
Santiago González Escudero, «Semántica e inferencia en la controversia estoicos-epicúreos», fue un escueto pero brillante análisis filológico de la disputa entre el estoicismo y el epicureísmo, pugna en la que al logicismo terminologista de los miembros de la Stoa se vino a oponer el sensismo del Jardín. José V. Peña Calvo abordó, en la suya, la utilidad heurística que el concepto de fracaso escolar pueda tener en la investigación pedagógica; rechazando las connotaciones psicológicas de tal concepto, reivindicó la validez de algunos conceptos alternativos, teñidos de preocupaciones sociales más intensas, como el de mortalidad escolar.

5. La sesión matinal del 27 de septiembre, dedicada también monográficamente a las matemáticas, se abrió con la ponencia de Javier de Lorenzo, matemático y filósofo de la matemática, bien conocido en España por sus ensayos sobre la lógica y sobre el método matemático. El propio título, «La matemática, ¿mero lenguaje?», denota la problematicidad de su planteamiento, al tiempo que pone al descubierto una de las más antiguas cuestiones acerca de la matemática: la de si ésta es una ciencia con objeto propio o si, por el contrario, se trata tan sólo de un instrumento formal para el desenvolvimiento de otras ciencias con un objeto material definido.

En la tradición moderna de la filosofía de la ciencia, arraigada en la figura de Galileo, la matemática ha venido considerándose como el lenguaje fundamental de las ciencias. Se ha forjado, en torno a esto, un extendidísimo lugar común consistente en tomar a la matemática como un lenguaje imprescindible y de aplicación general a todas las demás ciencias. El mismo Poincaré, caracterizando a las matemáticas como el arte de conceder un mismo nombre a las cosas distintas, ha sido destacado impulsor de ese enfoque -estrictamente lingüístico, que desatiende todo contenido conceptual- que se ha consagrado a elaborar un lenguaje semejante, en el que los aspectos sintácticos tienen una presencia omnímoda.

Sin embargo, Javier de Lorenzo cree que la matemática no es solamente un lenguaje. En primer término porque los conceptos van ligados a las expresiones, a las fórmulas, y de ese modo determinan las operaciones, el cálculo. En segundo término porque la matemática está ejerciendo diversás influencias: determinando una cierta manera de percibir la realidad, condicionando una forma específica de hacer ciencia y, en fin, proporcionando una implícita concepción del mundo que excede con mucho el simple marco de la actividad científica. Como ejemplo, se aduce el espacio métrico euclídeo, que lleva a aceptar algunas propiedades (homogeneidad, ilimitación, etc.) que modifican, al no coincidir con ella, nuestra percepción limitada del espacio... 
De las dos concepciones que hasta ahora se han examinado, la de la ciencia matemática como algoritmo, y la de las matemáticas como raíz de una cosmovisión, ninguna muestra que se trate de un simple lenguaje, ni siquiera la primera de ellas. Pues existen, en efecto, unos contextos de descubrimiento que no se explicitan lingüísticamente (no siempre los datos lingüísticamente expresados son suficientes para alcanzar la solución de un problema). Además, la ejecución de operaciones matemáticas comporta no sólo procedimientos de cálculo sino, especialmente, una cierta forma de razonar; $y$, como señaló el ponente citando la dialéctica del «Menón» platónico, de la forma de razonar que se emplee dependerán las conclusiones.

Dicho de otro modo, el tipo de geometría que se adopte determinará la clase de ciencia física que se haga. De Lorenzo denominó, a esas cosmovisiones en cuya creación tiene tanta importancia la matemática, «burbujas conceptuales». Tales burbujas orientan nuestra concepción de la naturaleza de forma peculiar, y en ocasiones incluso contra lo que nuestra percepción de lo real nos suministra: piénsese en el espacio natural, por un lado, y en la diversa representación de las figuras de la pintura bizantina (plana) y la renacentista (perspectiva, en punto de fuga).

En suma, la matemática es un modo de conceptualizar, y no algo verdadero o falso. No se trata de pensar que la naturaleza esté matemáticamente articulada, sino de comprender que la matemática sirve para aprehender algunas articulaciones de la naturaleza. No puede, por tanto, admitirse la ingenua reducción de las matemáticas al plano de lo meramente lingüístico.

El vicepresidente de la Sociedad Española de Historia de las Ciencias, Mariano Hormigón Blánquez, leyó una ponencia dedicada al fenómeno histórico del bourbakismo: «Las matemáticas: un forzoso matrimonio de contenidos y notaciones».

La tesis del ponente viene contenida en el título, pues entiende que el matemático es un lenguaje que no puede quedar reducido a lo puramente sígnico. Creemos que también él habría de contestar negativamente a la pregunta, hecha por Javier de Lorenzo, sobre si la matemática es meramente un lenguaje. Ya que, a su parecer, en ninguna ciencia tanto como en las matemáticas los aspectos formales, lingüísticos, han ejercido influencias tan señaladas sobre los contenidos.

Esta tesis, no obstante, difiere de los grandes principios que han servido de base a la difusión de un formalismo a ultranza durante este siglo. En el año 1940 se editaron los «Elementos de matemáticas» con la firma de Nicolás Bourbaki. Éste, como es sabido, no es sino el pseudónimo (misterioso en sus indicios) de un colectivo de matemáticos. Por si este rasgo no fuera ya de sobra excepcional en la historia de la ciencia pueden añadirse otros: haber esta- 
do compuesto por matemáticos muy importantes, renovarse continuamente (sus miembros raramente han pasado en él de los cincuenta años de edad), y tener un carácter abierto para promover la discusión crítica de todas las publicaciones que desde esa fecha han ido preparando. Los dos rasgos más significativos del bourbakismo son el axiomatismo formalista, y el rechazo de todo empirismo.

Para el historiador de la ciencia, el surgimiento del grupo Bourbaki tiene una doble raíz. Una, de índole teórica, está en la necesidad de reivindicar la función científica de la teoría, de potenciar el papel de los matemáticos en el panorama de la ciencia. Otra, inmersa en lo socio-político, prende en el deseo de recuperar el prestigio de la ciencia francesa en la posguerra; no debe olvidarse que el nacimiento del colectivo coincide en el tiempo con la transferencia, desde Alemania hacia Francia, de la problemática axiomatizadora de D. Hilbert.

Por lo que a la fundamentación de la matemática respecta, basta a Bourbaki con admitir — véase la opinión de Alberto Dou reseñada antes- que puede fundarse en una reformulación de la lógica clásica y en la teoría de conjuntos. La aparición del grupo tuvo pronto el respaldo de la utilidad práctica del formalismo, aun cuando su creatividad fuese escasa (René Thom, a quien Hormigón cataloga como platónico, critica a Bourbaki por no haber producido ni un solo teorema original).

Para Bourbaki, lo que distingue a un texto matemático es su formalización, y la tarea del formalista deberá ser exclusivamente la construcción de un método axiomático. Decimos que en exclusiva porque se pretende construir una matemática que no esté contaminada por factores políticos o reales externos. Los matemáticos deben aplicarse a sus fórmulas y desentenderse de cualquier utilidad, de toda posible aplicación externa - ni siquiera a las ciencias físicas - de los hallazgos de la matemática. En suma, se trata de evitar, como ya pretendiera Hilbert, cualquier contacto con la realidad empírica (fenómeno muy similar, pues, al de los estructuralismos lingüístico y antropológico).

Concluyendo: el bourbakismo, con su proyecto axiomático y antiempírico, ha extendido sus influencias por todas partes, incluso en los primeros niveles de enseñanza: la geometría clásica ha sido reemplazada por un axiomatismo casi dogmático, y las matemáticas se han encerrado en su propio lenguaje. No obstante, y sin que ello suponga recuperar las posiciones del platonismo o las del intuicionismo, debe reconocerse que hay muchos problemas que son irreductibles a estructuras meramente formales.

Para la tarde se programó una mesa redonda sobre «Psicología de la inteligencia verbal: problemas y métodos», mesa que presidió José Muñiz Fernández — próximo a Mariano Yela, probablemente el psicólogo español más 
conocido fuera de nuestras fronteras-y en la que tomaron parte varios jóvenes investigadores españoles. No es nuestro propósito aquí la descalificación, pues es innegable el interés que encierran los experimentos que, sobre inteligencia verbal, refirieron los componentes de la mesa; pero es preciso aclarar que las intervenciones producidas apenas tenían lugar oportuno en un congreso, como el que reseñamos, consagrado a la teoría de las ciencias.

Es de celebrar, no obstante, la inquietud que acreditaron los comunicantes por buscar alternativas metodológicas a una psicología demasiado aferrada - hasta el presente- a la estadística. Acaso una reunión de psicólogos hubiera sido mejor marco para hacer públicos los trabajos que se están realizando en inteligencia verbal; pero no deja de ser encomiable el esfuerzo de nuevas generaciones de psicólogos por responder a cuestiones profundas (como, por ejemplo, qué se entiende por inteligencia verbal, o por qué unos individuos la poseen en mayor medida que otros), en vez de contentarse con la acumulación de datos inanes que el análisis factorial y la psicometría han venido aportando hasta hace unos veinte años.

De las comunicaciones, últimas de este congreso, cabe destacar un análisis, hecho por Ildefonso Murillo, de la epistemología de Hans Albert; y un serio - pero difícilmente demostrable - nexo entre la concepción de la teoría de la ciencia de Ortega y Gasset, y la de Popper, propuesto por Antonio Revilla.

Rosa Rodríguez Ladreda, en una comunicación sobre la epistemología y su método apuntó muy certeramente a las grandezas (?) y miserias de esa disciplina en tanto que sustituta de la filosofía. La corriente filosófica que más hizo para generalizar las reflexiones sobre la ciencia, el positivismo lógico, ha sido engullido también por la historia: a pesar de la intachable coherencia y rigor crítico de los miembros del Círculo de Viena, debe reconocerse hoy que la crisis de la racionalidad científica, denunciada por los positivistas, ha sido al propio tiempo una crisis de la pretendida analiticidad de la filosofía. La filosofía ha perdido, acaso, el supuesto poder de decretar la racionalidad o irracionalidad de las ciencias.

6. Gustavo Bueno, que como se sabe es el gran animador de esta original revivificación de la teoría de la ciencia en nuestro país, tuvo a su cargo la última ponencia de este congreso, dedicada al «Lenguaje geométrico y física en Newton», a la que siguió el acto de clausura de una convención tan atractiva como prolongada y agotadora.

Un curioso bucle hizo que el congreso, que se había inaugurado con la invocación de $\mathrm{R}$. Thom a la causalidad de Aristóteles, se cerrase con la reivindi- 
cación de otro de los pilares del sistema aristotélico: el hilemorfismo. Al entender de Gustavo Bueno, hacer gnoseología es tomar como punto de partida -antes que el hiato sujeto/objeto- la distinción entre forma y materia.

Atendiendo al tema general del congreso, Bueno hizo una clasificación de las teorías científicas - desde su conocida doctrina del cierre categorial-con arreglo a los tres grandes ejes que la división de funciones lingüísticas de $\mathrm{Ch}$. W. Morris contempla: sintáctico (términos, operaciones y relaciones), semántico (fenómenos, procesos y estructuras), y pragmático (ontologismos, dialogismos y normas).

De acuerdo, pues, con esto, y puesto que el lenguaje es una forma de las ciencias, si bien no exclusivamente, se precisa esa clasificación de teorías. El descripcionismo prima la dimensión semántico-pragmática del lenguaje, minusvalorando la forma en favor de la materia. El teoreticismo se centra en las funciones sintáctica y pragmática, marginando los hechos (p. ej. Popper). El adecuacionismo atiende a la dimensión sintáctico-semántica (v. gr. Tarski), conectando materia y forma. El materialismo gnoseológico adoptado por Bueno funde, finalmente, las tres dimensiones lingüísticas.

Desde estos presupuestos conceptuales, se abordó un conocido caso histórico: la deducción geométrica que Newton hizo de la ley de las áreas. El ejemplo, tan famoso y repetido, sirvió para un brillante análisis gnoseológico del ponente. Según éste, la labor realizada por Newton no sería fruto de una genialidad intuitiva, como se ha glosado abundantemente, sino una minuciosa actividad constructiva para enlazar un descubrimiento geométrico de Euclides con el hallazgo kepleriano de las regularidades orbitales.

Para Bueno, cada tipo de teorías citado explicaría de un modo propio el caso histórico propuesto. Los descripcionistas entenderían a Newton como mero repetidor de los hechos desvelados por Kepler. Para los teoreticistas, la expresión geométrica newtoniana es independiente de la realidad empírica que el teorema explica. Los adecuacionistas, en último término, conciben como correspondientes el orden real y el ideal, es decir, la forma geométrica del teorema se entendería como paralela a la realidad física material.

Frente a todas esas posibles interpretaciones, que considera insuficientes, el materialismo gnoseológico recoge tan sólo parcialmente ese conjunto de teorias. Negado cualquier posible isomorfismo entre el plano geométrico y el plano físico, hay que proclamar la peculiaridad de la figura geométrica: el lenguaje, la forma, encierran algo diferente de la realidad física o materia. Para Gustavo Bueno, la verdad (objeto central de cualquier gnoseología) no consiste en la correspondencia entre la forma y el fenómeno físico (como el adecuacionismo quiere), ni en hallar la cosa misma (según el descripcionismo interpreta), ni tampoco la pura formalidad (que el teoreticismo ensalza): la verdad, 
interpretando la reformulación newtoniana de Kepler, debe entenderse como una unidad sintética. Es decir, se formaría con las relaciones de identidad que hay entre los planetas, descritos por Kepler-Newton, y sus fases, en la medida en que se coordinan aspectos sintácticos, semánticos y pragmáticos.

En definitiva, la formulación geométrica de Newton (tomada de Euclides) no es un mero canal lingüístico para encauzar una teoría física moderna desde un teorema geométrico antiguo. En palabras de Bueno, las áreas barridas por los planetas keplerianos en sus órbitas son algo así como las sombras platónicas de las relaciones geométricas euclidianas.

La más profunda cuestión, acaso, de la filosofía del lenguaje -el presunto isomorfismo lenguaje/realidad - viene a ser reemplazada por otra distinta: puede que se dé un isomorfismo entre varios lenguajes de etapas distintas de la física (el euclidiano, el kepleriano, el newtoniano, etc.).

Antes de completar esta reseña del tercer congreso de teoría y metodología de las ciencias, que esperamos tenga continuidad en otros posteriores - se perfila ya uno para el año 1987, con sociología como gran tema posible-, hemos de aclarar que han quedado sin consignar buena parte de las comunicaciones presentadas; mencionamos por ello, al menos, la presencia de Camilo Cela Conde, Antonio González Carlomán, Jaime Arnau, Juan Bautista Fuentes, José $\mathrm{M}^{\text {a }}$ García Prada, y tantos otros que en número próximo al centenar contribuyeron a mantener el magnífico tono del congreso.

Juan A. CANAL

Oviedo, 1986 